
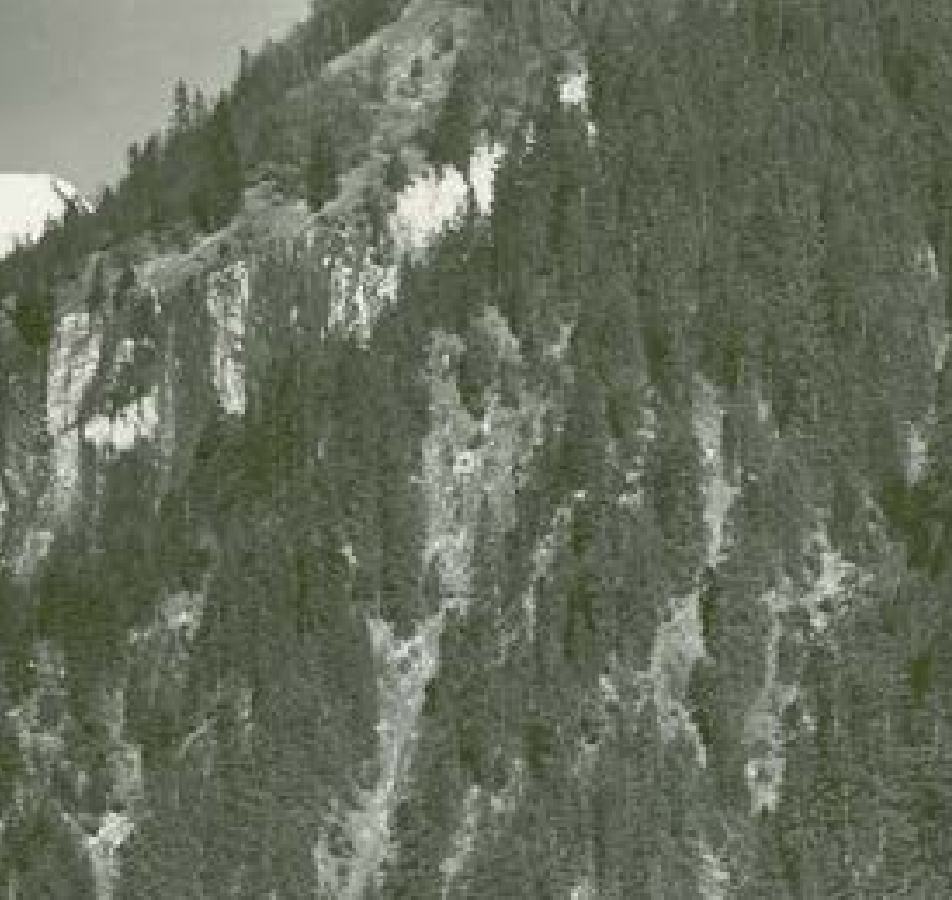

is

T.
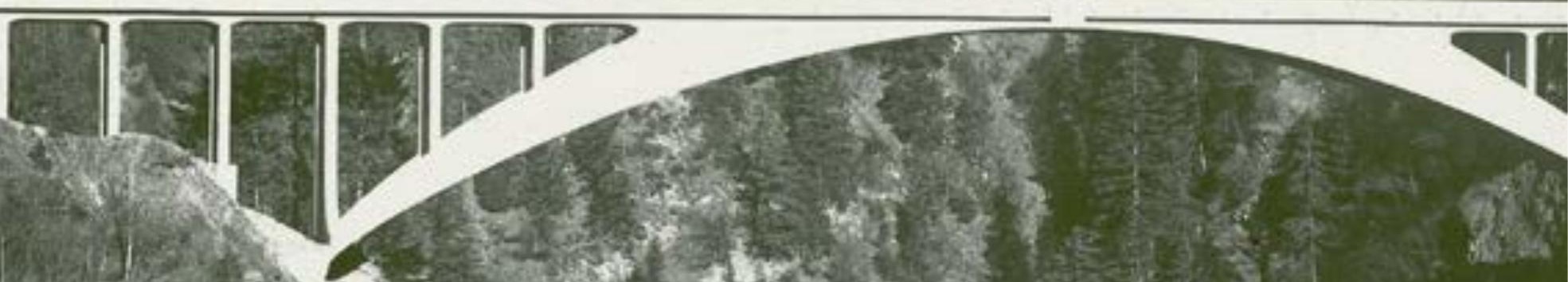

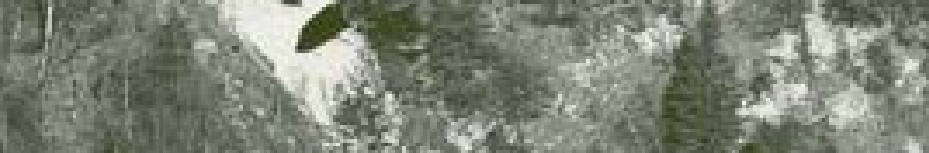

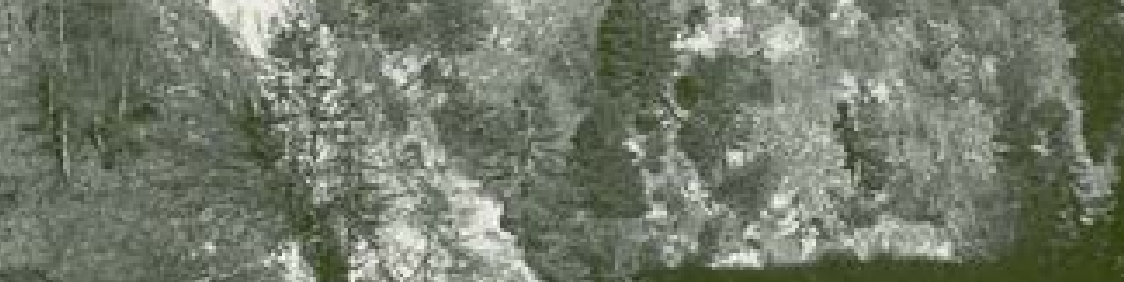

M.

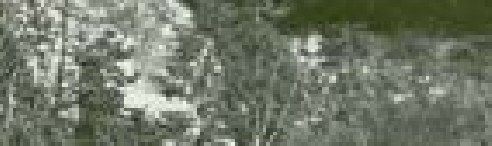

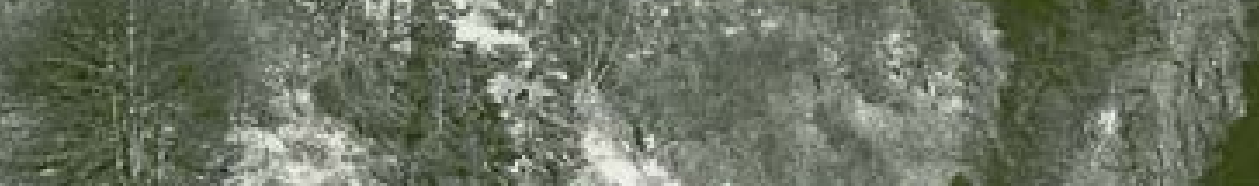

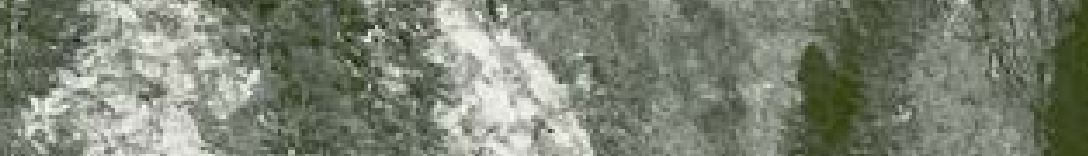

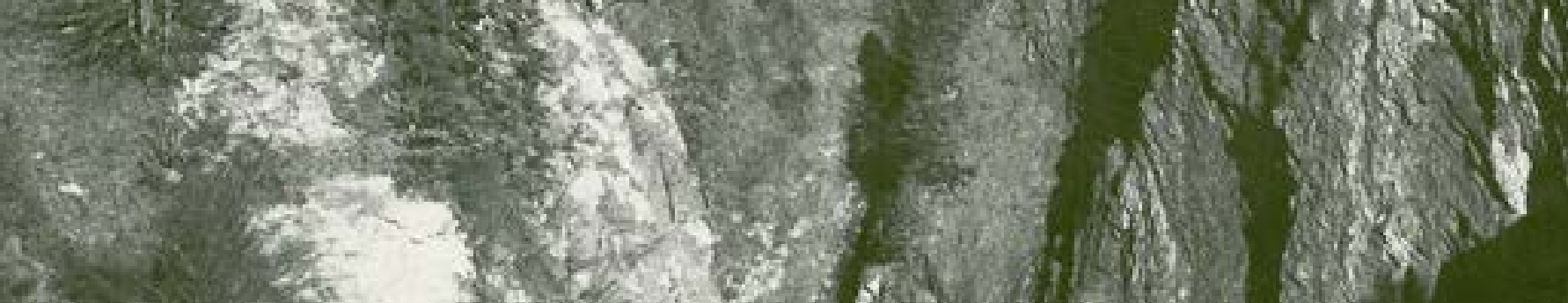

s. at. Q

(3)

3if Wets: (2)

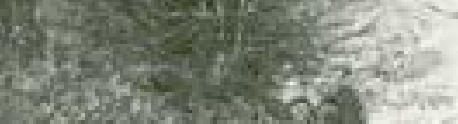

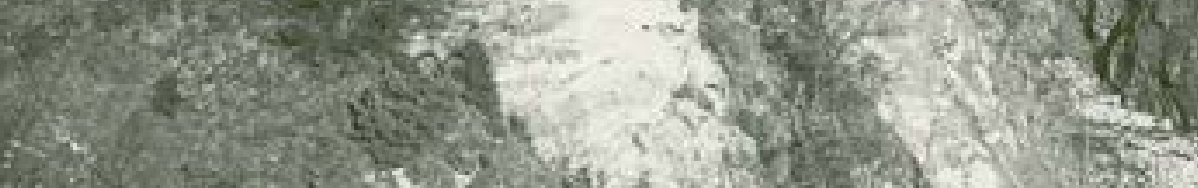

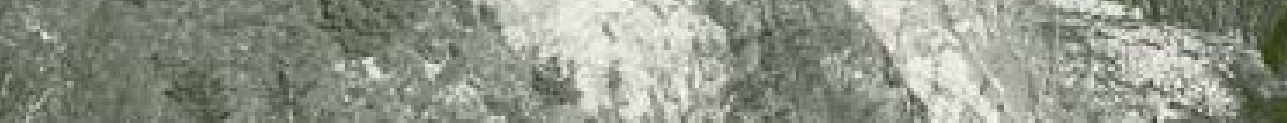

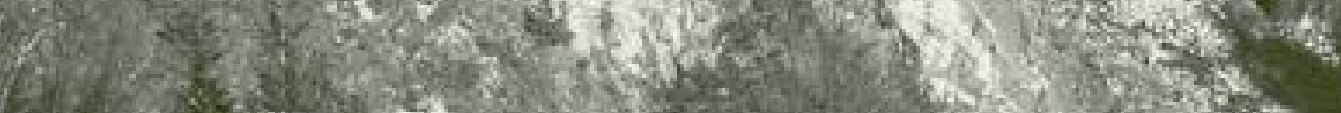

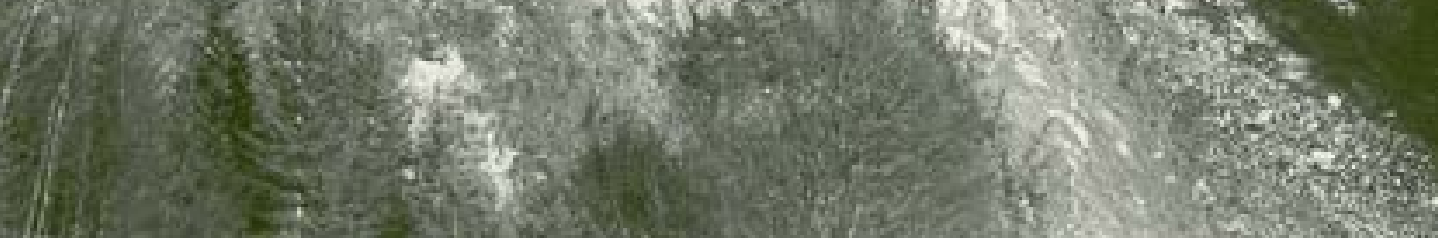




\title{
Secolarizzazione, memoria e nuovi revivals: la difficile riscrittura del presente
}

\author{
Secularization, memory and new revivals: the difficult rewriting \\ of the present
}

\begin{abstract}
The architectural project of re-use, in a context full of historical, cultural and environmental values such as the Alpine one, must inevitably confront with the list of conceptualizations elaborated during the twentieth-century modernity.

First of all we have to think about memory which is the main raw material of architecture and its history, a theme on which the culture of restoration has been built, and on which the rhetoric of re-use has been shaped in recent times, with the gradual addition of other contemporaneity values such as soil consumption, sustainability, zero growth.

The theme of memory seems in some cases to negotiate the one of secularization, a term that often comes to coincide with modernity.

The modernity that has standardized and flattened the Alpine territory, raising questions about the meaning of the "encounter", its ability to question prejudices, beliefs, and consolidated rhetorics. The "encounter" that takes place in the moment in which the contemporary urban culture succeeds in crossing landscape and Alpine architecture issues, creating disorientation, reopening the eyes of the observer and rebooting history by questioning the present.

The "rewriting of the existent" therefore raises issues that are difficult to make explicit, such as the recognition and critical knowledge that are the basis of design responsibility. This is accompanied by the threat caused by a prescriptive approach which, in light of the aspects of control and of technological and environmental management of the mountain context, can lead to the drift of the practice which must be avoided only through the assumption of risk linked to the design itself.
\end{abstract}

\section{Carlo Olmo}

Dean of the Faculty of Architecture at the Polytechnic of Turin from 2000 to 2007. He has taught at the École des Hautes Études en Sciences Sociales in Paris, at MIT in Boston and many other foreign universities. $\mathrm{He}$ is the author of numerous books, including the recent Città e democrazia (Donzelli, 2018), Architettura e storia (Donzelli, 2013), Architettura e Novecento (Donzelli, 2010).
Keywords

History, heritage, architecture, memory, norm. 
In un mondo in cui le parole smarriscono spesso il loro significato e rimangono senza padrone, quello che Hegel chiama "il lavoro del concetto" (Hegel, 1807) appare sempre più imprescindibile e purtroppo raro. Le parole sono utili per restituire un'immagine della realtà, ma quest'immagine è continuamente condizionata da chi il concetto lo utilizza. Ma quando, come nel caso della memoria, chi usa questa parola smarrisce il suo significato e la fa diventare una parola contenitore, il lavoro del concetto appare quasi impossibile. Nel gioco dialettico tra teoria e prassi di cui vive la parola, può accadere infatti che sia la prassi a prevalere, rendendo la parola asservita ad un potere e facendola così diventare il migliore veicolo di una fede: diventare una parola d'ordine. E alla memoria, ad esempio, accade sempre più sovente.

Paul Ricoeur nel suo testo più importante, La mémoire, l'histoire, l'oublie (Ricoeur, 2000) individua tre forme di memoria condizionata dalla prassi: la mémoire manipulée, la mémoire commandée, e la mémoire istitutionalisée. Nel jeu d'échange tra riuso e riciclo intorno ai temi del patrimonio e della patrimonializzazione che questo numero della rivista propone, sembrano poter entrare tutte e tre queste forme di memoria. Ł̀ difficile anzi che un riuso non le intrecci tutte e tre, come il riciclo possa non ricadere in una manipolazione della memoria. È possibile, a meno che non si avvii un autentico lavoro sulla parola.

La memoria costituisce la materia prima dell'architettura e della sua storia, e non è necessario attendere i revivals o i neo (classico, gotico, romanico o le diverse forme di postmodernismo). La memoria viene istituzionalizzata sin da Vitruvio e il rapporto tra forma e norma è molto più antico e rilevante di quello tra forma e funzione. Basta riprendere il filo del ragionamento che Aristotele mette in bocca a Ippodamo e che si istituzionalizza nella griglia, per capire quanto la Politica non debba aspettare l'omonimo testo del filosofo di Stagira per sancire come fondativo per l'architettura il nodo forma-norma (Laurenti, 2007). E questo ben prima delle regole che le città di nuova fondazione si porteranno dietro dall'antichità alle new towns scozzesi del secondo dopoguerra.
Ma nelle retoriche del riuso - che non a caso diventano dominanti con la crisi epistemologica all'inizio degli anni Settanta del XX secolo di un'altra parola chiave della cultura architettonica, il restauro -, come, e ancor più, in quelle ben più recenti del riciclo, entra soprattutto la mémoire commandée. Sono valori altri (il consumo del suolo, la sostenibilità, la crescita zero, che non a caso risalgono alla prima crisi energetica e al Club di Roma (Meadows, Meadows, Randers, Behrens III, 1972), o ancora la crisi di altre parole come diffusione o decentramento) a "reggere" la logica del riuso, sino a farlo divenire adattivo ad altre forme di bisogno (altro termine cardine della lingua degli architetti almeno dal secondo dopoguerra) (Olmo, 2010).

A rimettere in gioco un possibile lavoro del concetto è quello che Ryszard Kapuscinski mette alla base del riconoscimento dell'altro: l'incontro (Kapuscinski, 2007). Perché l'incontro ha questa possibilità? Perché l'incontro riapre i giochi, spaesa, rimette in moto la storia come interrogazione del presente (Bloch, 1949). Se infatti è vero che l'altro è alla base persino di una fondamentale alterità - il riconoscimento di valore della montagna rispetto alla pianura per non fare che l'esempio più semplice (De Rossi, 2014) -, lo spaesamento che l'incontro procura rispetto ai codici d'attesa impone il ripensamento delle narrazioni che strutturano le memorie comandate e istituzionalizzate nel caso studio che qui si tratta: il riuso delle architetture alpine e più in generale di tutta l'opera di costruzione storica del territorio montano. E tale spaesamento generato dall'incontro apre a un doppio lavoro sulla memoria: quello dello storico che deve prendere in mano insieme problemi e interrogativi che la memoria collettiva organizza e rielabora, e quello del progettista che non può affidarsi semplicemente alla sanzione dell'alterità che gli viene offerta.

La domanda che si pone Emma Bovary guardando fuori dalla finestra - device che François Jullien pone all'inizio del suo testo Una seconda vita (Jullien, 2017), il perché vivo - qui si trasforma in perché vivo diversamente: e non basta la sostituzione del termine società con quello di comunità, viste le evi- 
denti ambiguità che hanno reso queste due parole anch'esse quasi prive di potere. L'incontro con la montagna trasforma questa domanda per il progettista in perché progetto e devo progettare diversamente in ambiente alpino? Non è un caso che Kapuscinski e Jullien condividano testi costruiti sul "lavoro sul concetto" di alterità e sulle sue matrici Lacaniane (Lofts, Rosemann, 1994).

L'incontro è d'altronde alla base dello spaesamento che la montagna pone al viaggiatore, romanticamente, scientificamente, drammaticamente sin dai primi tentativi di appropriazione del cittadino o dello straniero, se si vogliono usare due riferimenti diversi, uno politico, l'altro filosofico (Schneider, 2011). Perché il senso profondo di una domanda che pare far compiere un passo all'indietro a una modernità che aveva quasi omologato, quando non standardizzato, il territorio alpino a quello urbano sta tutto nella natura interrogativa dell'incontro, nella sua capacità di mettere in discussione pregiudizi, credenze, in altre parole la mémoire commandée con cui si arriva all'incontro. Perché però l'incontro diventi una nuova "Ère du soupçon", riprendendo il saggio di Nathalie Sarraute del lontano 1950 (Sarraute, 1950), c'è un passaggio fondamentale da compiere.

La "riscrittura dell'esistente", la sua possibilità di manipolazione, pongono infatti un altro nodo tutt'altro che semplice da sviscerare: il rapporto tutt'altro che banale che lega una parola, secolarizzazione, con altri due termini chiave oggi della lingua architettonica, riconoscimento e conoscenza (critica). Con sullo sfondo un fantasma epistemologico che all'inizio degli anni duemila vide discutere tutte le scienze sociali intorno a questa questione (Pomata, Cerutti, 2001). Secolarizzazione, riconoscimento, conoscenza: tutti termini che con il contenuto di questo numero hanno davvero molto a che fare.

Secolarizzazione è quasi sinonimo di modernità (Lübbe, 1965), e appare spesso come il tentativo di costruire un ordine umano post sostanziale: la centralizzazione del potere, la conseguente burocratizzazione delle strutture amministrative, l'idea di un diritto limitato e razionale trovano, tra le espressioni più rilevanti di quella che Carl Schmitt chiama il "governo della legge" (Schmitt, 1984), non solo il diritto amministrativo che regge regole e regolamenti urbanistici, ma anche l'omologazione delle culture progettuali. Culture che hanno, nella quasi infinita letteratura sullo standard, insieme una scommessa e un buco nero della modernità architettonica (Sarraute, 1950). Senza un lavoro sul concetto di secolarizzazione poco capiremmo di quanto accade in ambito alpino, almeno dopo la prima guerra mondiale ed in maniera più accentuata dopo la seconda (De Rossi, 2016).
Ma senza una riflessione sulle sue radici, sull'ampia ricezione di questa parola in ambiti differenti (giuridico, amministrativo, architettonico, urbanistico), difficilmente se ne può uscire: e il nodo che si ripresenta è quello dell'ambiguo rapporto tra riconoscimento e conoscenza critica.

Non esiste forse esempio di secolarizzazione post sostanziale più evidente - per la natura stessa del "fatto" architettonico omologato dalla prassi dell'edilizia alpina dalla fine degli anni Cinquanta, sin quasi ad oggi. Nei suoi aspetti sostanziali questa secolarizzazione vive di un riconoscimento complesso, via via definito attraverso filtri che studi tipologici, manuali, cataloghi costruiscono dall'inizio degli anni Venti e che romanzi come La montagna incantata fanno passare da scenografie di vita quotidiana a sostanza della malattia che la modernità ha insita: perché la narrazione su quanto valgano i patti, nel nostro caso tra l'amusement et le territoire, si rivela semplicemente una narrazione rassicurante (Adorno, Horkheimer, 1947). Quando poi l'essor des images genererà anche l'eclisse della narrazione (Brilli, 2010), persino lo spessore storico del riconoscimento lascerà spazio a forme di riduzionismo che toccano insieme sia la stessa idea di progetto che la stratificazione di rappresentazioni su cui esso si fonda.

Perché il rapporto tra la memoria e la storia, e tra la memoria collettiva - anche in questo caso ci sono dei lavori fondamentali su ciò che è memoria collettiva e ciò che è memoria individuale, in primis quelli di Maurice Halbwachs - e le storie che sono costruite secondo molteplici e parziali punti di vista, non è certo banale o banalizzabile. Le argomentazioni che fanno parte di un patrimonio interpretativo sono infatti già esse stesse "una memoria", ma per orientare le pratiche devono poter atterrare su prove, su ciò che Ricoeur chiama le "prove documentarie". In discussione deve andare per primo lo statuto di una storia che si vuole critica, in grado di interrogare le diverse memorie. Le parole della storia per contare devono aver peso (Goody, 1986), mentre il linguaggio può ammalarsi (Wittgenstein, 1978) e generare una distorsione prima, e una migrazione di significati poi. Considerazione che vale ancor più quando, come nella scrittura storiografica contemporanea, entrano in gioco anche concetti etici o politici, che dalle parole dovrebbero discendere: entrano cioè in gioco les usages politiques du passé (Hartog, Revel, 2001) per legittimare tradizioni o identità locali o nazionali (Geremek, 2009), che nel caso dell'architettura alpina pongono un ulteriore problema, quello della traduzione e della trascrizione delle parole che dovrebbero restituire un fatto (Cerutti, Pomata, 2001). Traduzione e trascrizione che hanno sto- 
rie troppo complesse per essere in questa sede anche solo accennate.

Inoltre, se le parole che si utilizzano rimangono non discusse, non fanno altro che alimentare la costruzione di recinti, tanto più improbabili quanto più producono revisionismi - quando non revanscismi - nella costruzione dell'opinione pubblica, fondandosi proprio sul mancato rapporto, come si è detto, tra argomentazione e prova documentaria. E forse, ancor di più, si impoveriscono le rappresentazioni che sullo stratificarsi di significati di una parola si sono create negli anni, come nel caso dei trente glorieuses e della loro modernità precocemente consumata. L'esempio più immediato, ma forse anche questo da storicizzare, è l'uso del termine classico. Nessuna civiltà, neanche quella greca, si è davvero realizzata in modo "classico". La rappresentazione di ciò che è classico si fissa in un modello - la cui elaborazione può essere storicizzata - in gran parte non realizzato, che muta poi in qualcosa di metastorico. Un modello che perde di spessore quando le rappresentazioni che comunque con quella parola e la sua storia si misurano, diventano citazione della citazione, sino a contaminazioni che diventano alibi proprio per aggirare il problema dei tempi che un'architettura comunque incorpora.

La forte crisi di legittimità che l'architettura e la storia dell'architettura contemporanea oggi conoscono spesso nasce anche dalla disinvoltura - mi si consenta l'espressione - con cui oggi architetti e storici usano le parole. Rendendo la possibilità di costituirsi come critica della memoria collettiva su cui si opera quasi evanescente. E determinando - senza voler mutuare la parola - una histoire, dopo una mémoire, commandée. Può non esserlo se si riesce a trasformare la stratificazione di significati, immagini, retoriche che la memoria collettiva offre, e che l'essor des images ha oggi ancor più radicalizzato, in prove documentarie di una storia non narrata e che non ha necessità di fondare le sue ragioni su "catastrofi" altre (il cambiamento climatico, i nuovi valori di una società non consumistica, la necessità di non consumare più suolo, ancor più in aree, come quelle alpine, dove il suolo è una riserva ancor più limitata). Forse solo così il progetto può provare a rinascere, muovendo dalla rimeditazione del rapporto, per come oggi si esprime, tra forma e norma.

Il documento (che sia una rovina di un'architettura alpina storica o un paesaggio più o meno incontaminato) è una produzione sociale la cui lettura è condizionata dal punto di vista, dalla ricezione che ha avuto e dalla presa di distanza dell'osservatore. In quanto produzione sociale rivela le intenzionalità di chi lo ha prodotto (casa o infrastruttura, standard o oggetto che si vorrebbe unico). La costruzione di un punto di vista richiede il confronto con la storiografia non solo dell'oggetto ma della stessa osservazione. La presa di distanza implica la coscienza della natura delle ideologie cui il restauro-riuso ha spesso fatto ricorso: ad esempio il retour à l'origine è oggi per il progetto in territorio montano quasi l'altra faccia dell'enrichessement ipotizzato da Boltanski e Esquerre. Due sono gli spunti che a questo punto del nostro ragionamento quella riflessione sull'enrichessement ci restituisce: il valore come giustificazione del prezzo e il collezionismo come nuova forma di mercificazione selettiva (Boltanski, Esquerre, 2017). È indubbio che oggi manca una teoria del valore riferita alle pratiche del riuso in area alpina (Ortiz, 2017), come e ancor di più manca una riflessione sui rischi di un collezionismo fondato sulla rarità del bene e spesso sul potenziale retour à l'origine, politico o anche solo nostalgico degli oggetti come dei paesaggi alpini.

La potenzialità di un processo conoscitivo inteso come forma di interrogazione in grado non solo di sottrarsi ai pre-giudizi della memoria collettiva, ma anche di proporsi come fondamentale attrezzo per una progettazione non ripetitiva e neanche alla ricerca esacerbata dell'unicità, sta tutta nella capacità di sfuggire a teorie dei valori eterogenerate, ma anche al collezionismo, magari colto e raffinato, e a qualsiasi forma di retour à l'origine. Solo l'aver presente che la memoria collettiva non è né un cassetto degli attrezzi da cui pescare a seconda del punto di vista che si sceglie, né un deposito di immagini, ma un palinsesto da risignificare, giocando sulla polisemia che esso conserva proprio grazie alle diverse memorie che incorpora, può aiutare il progettista ad avviare il suo lavoro.

Perché il progetto ha come statuto, quasi obbligato, quella che Foucault chiamava microfisica del potere, ossia un rapporto non tra forma e funzione, ma - vale la pena ribadirlo - tra forma e norme. Oggi la stratificazione di norme e regole diversamente legittimate è tale che pensare di ricostruire un rapporto tra forme e norme, assolutamente necessario per altro, si configuri quasi come un'utopia. Ancor più quando a fronte dell'abuso prima della modernità come rottura con la storia, poi del recupero di tradizioni spesso e necessariamente inventate, poi come ricerca di legittimazione altre del progetto, ed infine come espressione di una tutela paesaggistica che è l'ultima estenuata figlia di un retour à l'origine che ha segnato persino le vicende del restauro del moderno, il progettista si presenta e rimane davvero nudo.

La modernità, con tutte le parodie che l'hanno sovente accompagnata, è stata l'ultima stagione di una microfisica del potere dettata dalla riflessione sull'architettura, sui sistemi di regole che la po- 
tevano o dovevano governare (spesso la modernità è stata prescrittiva), sul rapporto - a volte persino tenero - tra la ricerca di invarianti e l'insostenibilità della ripetizione. Quella stagione ha subito, più di altre e proprio per questi presupposti, mimesis persino volgari, che diventano autentici paradossi quando la "modernità va sotto tutela" (Olmo, 2010), quasi esistesse un'origine o una sostanza della modernità da tutelare.

La sua crisi non legalizza però il relativismo di ogni specie, accompagnato dalle più diverse forme di legittimazione, che arriva a teorizzare il ribaltamento dei suoi principi fondativi e il suo trasformarsi in collezionismo (d'autore, d'ambiente, di maniere, persino di tradizioni costruttive). Senza una microfisica del potere, il potere che si esprimerà sarà di altri: tecnologi della costruzione, fisici dell'atmosfera, ingegneri che si occupano di energie certamente rinnovabili o di dissesti idrogeologici. Ma difficilmente la stessa cultura e antropologia alpina che si vorrebbero tutelare esisterebbero senza una cultura del rischio, capace di aprire nuovi visioni, che infatti appare il nemico primo di quelle prese di posizione.

E un progetto senza rischi nasce non tanto morto, quanto omologo a standard e prescrizioni, nasce come figlio di quella burocrazia che l'antropologo americano David Graeber così pessimisticamente ci pone davanti quasi come destino (Graeber, 2016). Un destino fatto di ripetizioni ad libitum e di patrimonializzazioni quasi coatte e acritiche. Ma forse nessuna cultura come quella alpina storica ha saputo, forse per necessità, trovare un rapporto tra forma e norma che più abbia saputo sfuggire alla possibile trasformazione del potere in algoritmi, e degli algoritmi in tutele cristallizzate. Almeno sino ad oggi. Questo numero della rivista mostra forse uno spiraglio, alcune possibili vie, per uscire da uno stallo dalle movenze oramai quasi circolari, ponendo il tema di una inedita manipolazione innanzitutto di senso dei materiali del patrimonio.

\section{Bibliografia}

Hartog François, Baruch Marc-Olivier, Thomas Yan, Gaudard Pierres-Yves (1992), «Verité judiciaire, verité historique», in Le Débat, n. 102.

Adorno Theodor W., Horkheimer Max (1947), Dialettica dell'lluminismo, trad. it. Solmi Renato (1966), Einaudi, Torino.

Bloch Marc (1949), L'apologie pour l'Histoire ou Métier de l'historien, trad. it. Apologia della Storia o Mestiere di storico (1998), Einaudi, Torino.

Boltanski Luc, Esquerre Arnaud (2017), Enrichissement. Une critique de la marchandise, Gallimard, Paris.

Brilli Elisa (2010), «L'essor des images et l'éclipse du littéraire», in L'atelier du centre de recherches historiques - Revue électronique du $C R H$, n. 4.

De Rossi Antonio (2014), La costruzione delle Alpi. Immagini e scenari del pittoresco alpino (1773-1914), Donzelli, Roma.

De Rossi Antonio (2016), La costruzione delle Alpi. Il Novecento e il modernismo alpino (1917-2017), Donzelli, Roma.

Geremek Bronislaw (2009), «Common Memory and European Identity», in Swoboda Hannes e Wiersma Jan Marinus, Politics of the Past: The Use and Abuse of History, Renner Institut, Wien.

Goody Jack (1986), The Logic of Writing and the Organisation of Society, Cambridge University Press, Cambridge. Graeber David (2016), Burocrazia. Perché le regole ci perseguitano e ci rendono felici, II Saggiatore, Milano. Hartog François, Revel Jacques (a cura di) (2001), Les Usages Politiques du passé, Editions de l'École des Hautes Études en Sciences Sociales, Paris.

Hegel Georg Wilhelm (1807), trad. it. De Negri Enrico (1973), Filosofia dello Spirito, La Nuova Italia, Firenze.

Jullien François (2017), Una seconda vita, Feltrinelli, Milano.

Kapuscinski Ryszard (2007), L'altro, Feltrinelli, Milano.

Laurenti Renato (a cura di) (2007), Aristotele. Politica, Laterza, Bari-Roma.

Lofts Steve, Rosemann Philipp W. (1994), «Penser l'autre: psychanalyse lacanienne et philosophie», in Revue Philosophique de Louvain, n. 1, pp. 82 e sgg.

Lübbe Hermann (1965), La secolarizzazione: storia e analisi di un concetto, trad. it. Pioppi Paolo (1970), II Mulino, Bologna.

Meadows Donella H., Meadows Dennis L., Randers Jørgen, Behrens III William W. (1972), The Limits to Growth, Club di Roma.

Olmo Carlo (2010), «ll diritto alla città e la città dei diritti», in Olmo Carlo, Architettura e Novecento. Diritti, conflitti, valori, Donzelli, Roma, p. 29.

Olmo Carlo (2010), «Una modernità sotto tutela», in Olmo Carlo, Architettura e Novecento. Diritti, conflitti, valori, Donzelli, Roma, p. 89 e sgg.

Olmo Carlo (in corso di pubblicazione), «ll Punto di vista e la presa di distanza: esiste un'etica della ricerca?», in Lexicon n. 26/27.

Ortiz Pierre-Henry (2017), «Pour une sociologie des valeurs - entretien avec Nathalie Heinich», in www.nonfiction.fr.

Pomata Gianna, Cerutti Simona (2001), «Premessa», in Quaderni Storici, n. 3, pp. 647-664.

Ricoeur Paul (2000), La Mémoire, I'histoire, l'oubli, Editions du Seuil, Paris.

Sarraute Nathalie (1950), «L'ère du soupçon», in Temps Modernes, n. 2.

Schmitt Carl (1984), Scritti su Thomas Hobbes, trad. it. Galli Cesare, Giuffrè, Milano.

Schneider Arnd (2011), «Sull'Appropriazione. Un riesame critico del concetto e delle sue applicazioni nelle pratiche artistiche globali», in Antropologia, n. 13.

Wittgenstein Ludwig (1978), Della certezza, Einaudi, Torino. 